\title{
Early application of tail nerve electrical stimulation-induced walking training promotes locomotor recovery in rats with spinal cord injury
}

\author{
S-x Zhang ${ }^{1}$, F Huang ${ }^{1}$, M Gates ${ }^{1}, X_{\text {Shen }}^{2}$ and EG Holmberg ${ }^{1,3}$
}

Study design: This is a randomized controlled prospective trial with two parallel groups.

Objectives: The objective of this study was to determine whether early application of tail nerve electrical stimulation (TANES)-induced walking training can improve the locomotor function.

Setting: This study was conducted in SCS Research Center in Colorado, USA.

Methods: A contusion injury to spinal cord T10 was produced using the New York University impactor device with a 25 -mm height setting in female, adult Long-Evans rats. Injured rats were randomly divided into two groups ( $n=12$ per group). One group was subjected to TANES-induced walking training 2 weeks post injury, and the other group, as control, received no TANES-induced walking training. Restorations of behavior and conduction were assessed using the Basso, Beattie and Bresnahan open-field rating scale, horizontal ladder rung walking test and electrophysiological test (Hoffmann reflex).

Results: Early application of TANES-induced walking training significantly improved the recovery of locomotor function and benefited the restoration of Hoffmann reflex.

Conclusion: TANES-induced walking training is a useful method to promote locomotor recovery in rats with spinal cord injury. Spinal Cord (2016) 54, 942-946; doi:10.1038/sc.2016.30; published online 12 April 2016

\section{INTRODUCTION}

Currently, all theories concerning central mechanisms of walking refer to the central pattern generator (CPG) concept. ${ }^{1} \mathrm{CPG}$ is a network of spinal neurons that controls locomotion including walking, flying and swimming and is found in the spinal cord of all invertebrates, vertebrates and humans. ${ }^{2} \mathrm{CPG}$ generates patterns of rhythmic activity for locomotion, even in the absence of external feedback or supraspinal control, but normally it is modulated by supraspinal and peripheral inputs. ${ }^{1}$ Plasticity is an innate ability of neurons to rearrange their anatomical and functional connectivity in response to environmental input, and thus motor skills are acquired and maintained; activity-dependent plasticity is ubiquitous in the central nervous system. ${ }^{3}$

Walking training has become a commonly used strategy for locomotor recovery from spinal cord injury (SCI) both in animal experiments and in human clinical therapies, ${ }^{4,5}$ especially in the absence of normal supraspinal input, ${ }^{6}$ with body weight support walking and functional electrical stimulation having shown the potential to improve walking function in SCI victims. ${ }^{7}$ Recently, we developed a noninvasive technique, tail nerve electrical stimulation (TANES), which activates the CPG. We observed that once TANES was initiated the two paralyzed hind limbs of rats immediately become stronger, thus being able to support body weight and step and walk alternately, apparently indicating that the CPG has been activated and induced positive hind limb movement, as illustrated by walking with body weight support in SCI rats. TANES-induced walking has been used as a locomotor training method alone and by combining glial scar ablation and cell transplantation, significantly promoting locomotor recovery in rats with chronic SCI. ${ }^{8}$ Motor function improvement, most likely, is attributed to activity-dependent plasticity promoted by TANES through the activation of the CPG below the level of injury.

Evidence from animal experiments demonstrated that the rats' CPG may be located at the lumbar segments L1-2, and controlled by descending locomotor commands from the cortex and brainstem. ${ }^{9}$ Rats' CPG can be activated by TANES, resulting in the temporary body weight support walking in paralyzed subjects. Using TANESinduced walking as an open-field locomotor training paradigm, SCI rats showed significant improvement of motor function, indicating that the temporary ability to walk can be transformed into permanent ability to walk, as it relates to activity-dependent plasticity. ${ }^{8}$ To further investigate the mechanisms, universality and feasibility by which TANES results in the improvement of locomotor function, we started TANES-induced walking training on rats 2 weeks after SCI, without scar ablation nor cell transplantation in this study.

\section{MATERIALS AND METHODS}

Animal and SCI

Adult, female Long-Evans rats, with a body weight of 200-220 g, were used for this study. Animals were housed singly in plastic cages under standard

${ }^{1}$ Spinal Cord Society Research Center, Spinal Cord Society, Fort Collins, CO, USA; ${ }^{2}$ Department of Bioelectronics Nantong University, Nantong, Jiangsu, China and ${ }^{3}$ Department of Chemistry University of Alaska, Anchorage, AK, USA

Correspondence: Dr S-x Zhang, Spinal Cord Society Research Center, Spinal Cord Society, 2301 Research Boulevard, Suite \#203, Fort Collins, C0 80526-1826, USA.

E-mail: szhang@qwestoffice.net or sxzhangp@hotmail.com

Received 11 February 2015; revised 7 January 2016; accepted 14 January 2016; published online 12 April 2016 
conditions with free access to drinking water and standard food. Under deep anesthesia with sodium pentobarbital (50 $\mathrm{mg} \mathrm{kg}^{-1}$, intraperitoneal), all rats were contused at thoracic segment T10 using the NYU (New York University) impactor device with a $25-\mathrm{mm}$ height setting. The test rats were randomly divided into two groups. One group of injured rats received TANES-induced walking training (SCI+TANES; $n=12$ ) 2 weeks after injury. Rats in the other group were used as control (SCI control; $n=12$ ), receiving no TANES-induced walking training to determine the basal line of endogenous locomotor recovery curve. All rats were kept until 22 weeks after injury.

To relieve the pain, buprenorphine $\left(0.02 \mathrm{mg} \mathrm{kg}^{-1}\right.$, s.c., b.i.d. (subcutaneously, two times a day)) was used after surgery and the usage was continued for at least 2 days, and thus analgesia was provided during the recovery phase. To prevent possible infection in the wound and bladder, antibiotic cephazolin (20 $\mathrm{mg} \mathrm{kg}^{-1}$, s.c., b.i.d.) was used for 1 week. Urinary bladders were carefully emptied by hand twice a day until adequate spontaneous voiding returned. In addition, saline solution $(0.9 \%, 10 \mathrm{ml}$, s.c.) was administered daily for 1 week to prevent dehydration. The body weight of animals was checked daily during the first 2 weeks after injury and weekly afterward.

All surgical and locomotor training procedures, including interventions, presurgery and postsurgery care, electrical stimulation and electrophysiological tests, were approved by the Institutional Animal Use and Care Committee of CARE Research, Inc. and are consistent with the Guide for the Care and Use of Laboratory Animals (1996).

\section{TANES-induced walking training}

TANES-induced walking training started 2 weeks after SCI. A physical therapy instrument (Type J18A1, Quan-Ri-Kang Company, Beijing, China) was used for the electrical stimulation. This instrument, with an automatically controlled middle frequency, has been demonstrated to produce neither side effects nor risks when being used in the clinic and at home (http://www.quanrikang.net/ Aboutus.asp? Title $=\% \mathrm{C} 6 \% \mathrm{~F} 3 \% \mathrm{D} 2 \% \mathrm{~B} 5 \% \mathrm{BC} \% \mathrm{~F} 2 \% \mathrm{BD} \% \mathrm{E} 9)$. It has a middle frequency carrier wave of $2.5-8 \mathrm{kHz}$ and low adjustable frequency of $0-150 \mathrm{~Hz}$ with a maximum output current of $100 \mathrm{~mA}$. The stimulating strength was adjusted according to rats' response to the stimulation, usually between 10 and $40 \mathrm{~mA}$ at a frequency of $4 \mathrm{kHz}$. TANES was performed as described previously. ${ }^{10}$ Briefly, the rats without anesthesia were placed individually in a plastic basin with a smooth, nonslip floor and a diameter of $120 \mathrm{~cm}$. The rat's tail was clamped by two electrodes connecting to the instrument (Supplementary Figure S1). Once the stimulating current entered the rat, its two hind limbs started to move extensively and alternatively. Thus, the rat was able to stand up, step and freely walk with body weight support around the open field (plastic basin) during the stimulation (Supplementary Video). To prevent possible muscle fatigue because of excessive contraction of the leg muscles, the moving frequency of the hind limb was limited to 40-60 steps per minute by modulating the stimulating strength. The walking training lasted 20 min per session, five sessions per week, for a total of 20 weeks. Thus, the total number of steps per training session was $800-1200$ with an average of 1000 .

\section{Evaluation of locomotor function}

BBB open-field locomotion rating scale. The behavioral outcomes were evaluated by two observers blinded to treatment group using the well-known Basso, Beattie and Bresnahan (BBB) open-field locomotion rating scale. ${ }^{11}$ After SCI, the BBB scores were collected day 1 , day 3 and day 7 post injury, and once a week afterward until the end of the experiment (22 weeks after injury). In addition, a video record (1-2 min long each time) was made weekly for each individual test rat. The moving patterns of rats' hind limbs were reviewed repeatedly and carefully with the help of slow-motion video records. The final BBB scores for each time point were determined by combining the BBB paper record with the results of the video record review. ${ }^{8}$

Horizontal ladder rung walking test. A horizontal ladder rung walking test was used to assess walking ability and to measure both fore limb and hind limb placement, stepping and inter-limb coordination. This test has been shown to be sensitive to chronic movement deficits after lesions to the motor system, including rat models of stroke and SCI. ${ }^{12}$ The apparatus (Columbus
Instruments, Columbus, OH, USA) used for this experiment consists of two side walls made of Plexiglass ( $9 \mathrm{~mm}$ in thickness) and 47 metal rungs $(3 \mathrm{~mm}$ in diameter), which were inserted into the side walls to create a floor with irregular distances $(8-30 \mathrm{~mm})$ between any two rungs. The side walls are $100 \mathrm{~cm}$ long and $21 \mathrm{~cm}$ high measured from the height of the rungs. The space between the two side walls is $55 \mathrm{~mm}$. During the tests, rats were allowed to walk across the horizontal ladder from one end to the other. A video camera was positioned at the level of rungs, and thus positions of all four limbs could be recorded simultaneously. The video recordings were analyzed using frameby-frame analysis. ${ }^{12}$

All rats were trained to cross the ladder by first walking on the corrugated surface of a glass board covering the rungs. Before the test, no animals practiced walking on the rungs, thus preventing them from learning the pattern beforehand. ${ }^{12} \mathrm{~A}$ footfall error is defined as the misplacement of the animal's foot, such that the hind paw to ankle falls completely below the level of the rungs (Figure 2a) rather than being placed onto the rungs. ${ }^{13}$ The number of footfall errors and the number of steps were counted separately for each hind limb and presented as a ratio of errors. Each rat was allowed to walk on the rungs three times, and the ratio of errors per step was averaged as the error rate for the rat tested. ${ }^{12}$

\section{Hoffmann reflex}

Hoffmann reflex (H-reflex) was measured to assess the neurophysiological recovery of spinal reflex modulation in this study. The instrument used for electrophysiological tests contains two parts, the Sierra Wave System (Cadwell Inc., Kennewick, WA, USA) and a Magstim $200^{2}$ stimulator with a 50-mm coil (Magstim, Whitland, UK). Disposable Sub-dermal Needle Electrodes $(13 \times 0.40 \mathrm{~mm}$ stainless steel needle; Technomed Europe, Beek, The Netherlands) were used as recording, reference and ground electrodes for all electrophysiological tests. The interelectrode impedances were kept $\leqslant 2.5 \mathrm{k} \Omega$. Electrophysiological tests were carried out on nonanesthetized, restrained rats ${ }^{10}$ before injury, and at 2 and 22 weeks after SCI. During the test performance, an assistant gently held the nonanesthetized rat with one hand and calmed the subject with another hand by slightly stroking if necessary.

To record the H-reflex in the test rats, the recording needle electrode was inserted into the belly of gastrocnemius muscle in the hind limb. The reference electrode was placed into the muscle tendon. The ground electrode was placed subcutaneously near the tail root. ${ }^{14}$ The magnetic stimulating coil was placed over the L4 4 roots of the spinal nerve, and the nerve was stimulated with $35 \%$ of maximum strength of magnetic stimulation. In each test, 10-15 traces in both left and right sides were recorded. For the data analysis of the H-reflex test, the $\mathrm{H}$-wave latency, $\mathrm{H}$-wave amplitude, $\mathrm{M}$-wave latency and $\mathrm{M}$-wave amplitude were measured and collected with 10-15 traces for each side, and the $\mathrm{H} / \mathrm{M}$ amplitude ratio was calculated.

\section{Statistical analysis}

A power analysis was used to determine the sample size in this study. From our previous studies, the BBB score was $9.12 \pm 1.25$ (mean \pm s.d.) 6 weeks after the SCI. There was no significant change up to 22 weeks after injury. We hypothesized that the BBB score would rise 2 points, from 9.12 to 11.12 (that is, the locomotor function would improve, advancing from sweeping without body weight support to walking frequently with body weight support) at the end of this experiment with TANES-induced walking training. The standardized effect size (signal/noise ratio) was calculated by (mean 1 - mean 2)/s.d.: that is, in this study, $(11.12-9.12) / 1.25=1.6$. The sample size was determined for the experiment with two groups with an effect size of 1.6, a power of 0.9 , a significance level of 0.05 and a two-sided test. In a relative statistical table, the sample size was found to require 10 animals in each group. To guarantee the reliability of data, we added two more animals into each group, making the total sample size as 24 rats ( 12 rats per group) with an actual power of 0.95 . All quantitative data were expressed as mean \pm s.e.m. BBB scores for the hind limbs from the same group were averaged to yield one score for each time point. Because there were only two groups with small sample size $(n<30)$ in this study, two-sample $t$-test was used for comparison of two means. The BBB scores were compared separately at each time point with a two-tailed test. The data of horizontal ladder rung walking test and $\mathrm{H}$-flexes were all statistically 
analyzed by two-sample $t$-test (one-tailed). In all cases, differences were considered significant if $P<0.05$.

\section{RESULTS}

\section{BBB open-field rating scale}

In the SCI control group, the BBB score showed little change from 6 weeks after injury to the end of this experiment (22 weeks). However, 1 week after receiving TANES-induced walking training, the $\mathrm{BBB}$ score of trained rats began to outperform that of untrained rats in the SCI Control group. From 12 weeks after injury, differences in BBB scores between the two groups became statistically significant $(P<0.05)$. By the end of the experiment, BBB scores of trained rats increased to $11.58 \pm 0.72$, compared with $9.12 \pm 0.56$ of the untrained rats $(P=0.012$; Figure 1$)$.

\section{Horizontal ladder rung walking tests}

Horizontal ladder rung walking tests were carried out before SCI and at 22 weeks after injury. The numbers of footfall error and steps of the hind limbs were counted separately, and then the ratio of footfall errors per step was calculated. Rats before SCI were able to cross the horizontal ladder quickly with a very low ratio of footfall errors $(5.93 \pm 0.08 \%, n=24)$. In contrast, SCI rats without training took a longer time and showed a very high ratio of step errors while crossing the horizontal ladder (SCI control: $71.20 \pm 3.28 \%$ ). However, the average ratio of footfall errors in rats receiving TANES-induced walking training was significantly reduced $(55.51 \pm 1.71 \%, P<0.001)$ when compared with the results of the untrained rats (Figure 2).

\section{Hoffmann reflex}

H-reflex information recorded is shown in Table 1. After SCI, there was an increase in $\mathrm{H}$-amplitude and a decrease in H-latency, M-latency and $\mathrm{M}$-amplitude. In this manner, the BBB scores dropped to zero immediately after SCI and then began to recover; the H-reflex also spontaneously recovered to some degree for the chronic duration. The H-reflex was significantly improved, as shown by decreasing the $\mathrm{H} / \mathrm{M}$ amplitude ratio in rats receiving TANES-induced walking training at the end of the experiment. In particular, the $\mathrm{H} / \mathrm{M}$ amplitude ratio in trained rats was significantly reduced $(0.2717 \pm 0.0200$; Table 1 , Figure 3$)$ compared with that of untrained rats $(0.3354 \pm 0.0288 ; P=0.045)$.

\section{DISCUSSION}

Results from the present study show that early application of TANESinduced walking training significantly promotes locomotor recovery in SCI rats. These findings indicate that TANES is a useful paradigm for walking training to promote locomotor recovery in rat models of subacute SCI. This sort of functional recovery may be partially associated with the tissue repairing and axonal regeneration, but, most likely, it is mainly attributed to the activity-dependent plasticity below the injury level of spinal cord resulting from locomotor training. These observations may be related to the activation of CPG triggered by TANES. On the other hand, nonspecific tail stimulation (manipulation of the tail, placing the electrodes) and/or just placing rats in an open field may induce the activation of CPG, therefore contributing to the locomotor recovery; however, this possibility is not great in our study.

CPG is a delicate network composed of a group of interneurons that generate movements and that contain the information necessary to activate different motor neurons in the appropriate sequence and intensity to generate motor pattern. ${ }^{15}$ In our rat model with contusion in T10, the CPG located in L1-2 should be intact in anatomy and function after SCI. It is our belief that during TANES-induced walking training, the CPG was being activated, all leg muscles were working and their signals were being conducted to the spinal cord. TANES may be similar to the sensory stimulation of epidura, which results in voluntary movement standing, and assisted stepping after motor complete paraplegia in a patient with SCI. ${ }^{16}$ Thus, repeated stepping and walking would result in the activity-dependent plasticity, which normally occurs in the spinal cord throughout life. Driven by input from the periphery and the brain, this plasticity has an important role in the acquisition and maintenance of motor skills and in the effects of SCI and other central nervous system disorders. ${ }^{17}$ In the present study, rats with walking training demonstrate significant locomotor recovery when compared with untrained rats; this effect could be the result of activity-dependent plasticity. In fact, this is probably a process in which temporary ability to walk transforms into permanent ability.

The horizontal ladder has been used as a stringent test of sensorimotor function, and performance of the task is disrupted following SCI. ${ }^{18}$ Our data demonstrate that TANES-induced walking training, which resulted in remarkable locomotor recovery, can also significantly reduce the deficits in hind limb sensorimotor function

\section{Functional Recovery after $\mathrm{SCl}$}

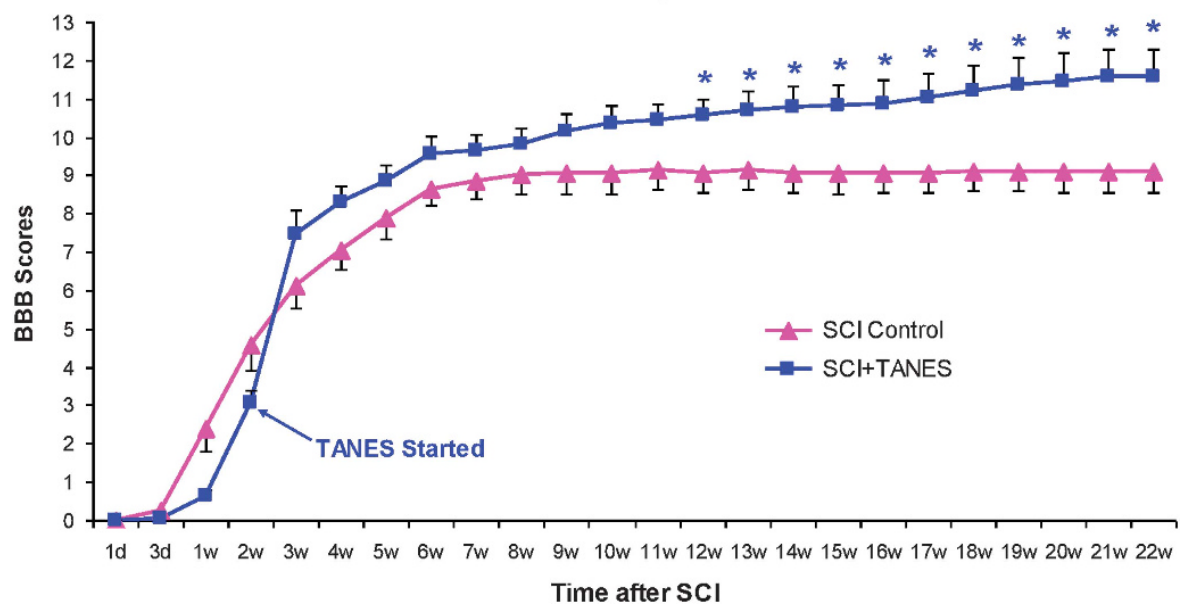

Figure 1 Locomotor recovery evaluated by BBB scale. The BBB score of trained rats ( $\mathrm{SCl}+\mathrm{TANES}$ ) was progressively higher than that of untrained rats (SCI control) from the third week. ${ }^{*} P<0.05$ indicates the significant difference in BBB scores between the two groups at varied time points. 


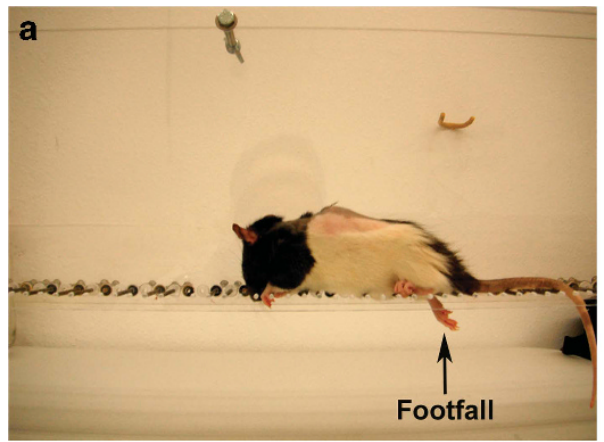

\section{b Horizontal Ladder Rung Walking Test}

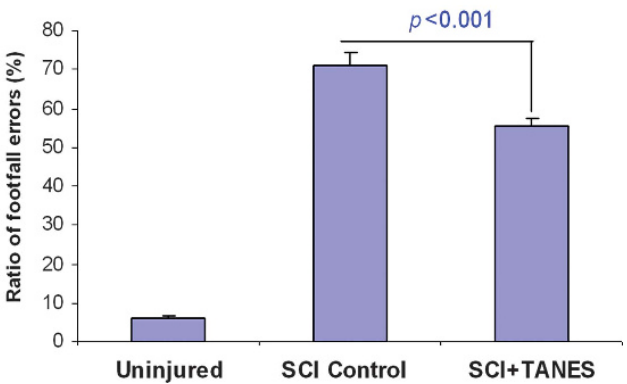

Figure 2 Horizontal ladder rung walking tests before $\mathrm{SCl}$ and 22 weeks after $\mathrm{SCl}$. A representative photo shows a footfall of rat's hind limb when walking on the ladder (a). The average ratio of footfall errors per step in rats with TANES-induced walking training (SCI+TANES: $55.51 \pm 1.71 \%$ ) was significantly lower than that in untrained rats (SCI control: $71.20 \pm 3.28 \%)($ b). Note: normal (uninjured) rats also have $5.93 \%$ footfall errors.

Table 1 Quantitative data of H-reflex before and after SCI

\begin{tabular}{|c|c|c|c|c|c|c|}
\hline & SCl time & H-Lat (ms) & M-Lat (ms) & $H-A m p(m V)$ & $M-A m p(m V)$ & $H / M$ ratio \\
\hline Normal $(n=24)$ & Uninjured & $7.69 \pm 0.07$ & $2.12 \pm 0.11$ & $3.44 \pm 0.15$ & $14.04 \pm 0.53$ & $0.2525 \pm 0.0103$ \\
\hline $\mathrm{SCl}$ control $(n=12)$ & 2 weeks & $4.99 \pm 0.10$ & $1.45 \pm 0.07$ & $4.72 \pm 0.26$ & $11.68 \pm 0.89$ & $0.4231 \pm 0.0305$ \\
\hline \multirow[t]{2}{*}{$\mathrm{SCl}+$ TANES $(n=12)$} & 2 weeks & $5.06 \pm 0.09$ & $1.32 \pm 0.07$ & $3.85 \pm 0.35$ & $9.97 \pm 0.91$ & $0.4075 \pm 0.0371$ \\
\hline & 22 weeks & $5.20 \pm 0.17$ & $1.41 \pm 0.15$ & $4.25 \pm 0.43$ & $15.89 \pm 1.43$ & $0.2717 \pm 0.0200^{*}$ \\
\hline
\end{tabular}

Abbreviations: Amp, amplitude; H, H-wave; H/M, H-wave amplitude versus M-wave amplitude; Lat, latency; M, M-wave; ms, millisecond; mV, millivolt; SCI, spinal cord injury; TANES, tail nerve electrical stimulation.

${ }^{*} P=0.045$, comparison of $\mathrm{H} / \mathrm{M}$ ratio between trained rats (SCI+TANES) and untrained rats (SCl control) 22 weeks after SCl.

Note: all data were expressed as mean + s.e.m.
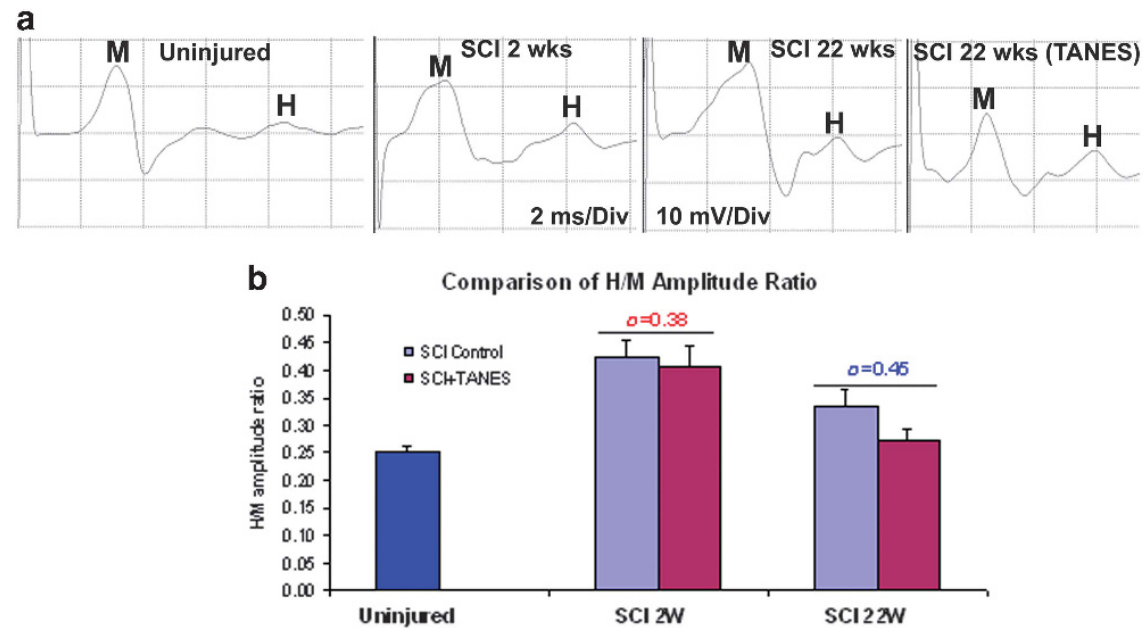

Figure $3 \mathrm{H}$-reflex test. (a) The representative wave forms of $\mathrm{H}$-reflex before (uninjured) and after $\mathrm{SCl}$. (b) After SCl, $\mathrm{H} / \mathrm{M}$ amplitude ratio increased (2 weeks) because of the increase in $\mathrm{H}$-amplitude and the decrease in $\mathrm{M}$-amplitude (Table 1) but slightly recovered later (22 weeks). Significant recovery of the $\mathrm{H} / \mathrm{M}$ amplitude ratio was found in trained rats (SCI+TANES, SCI 22 weeks), compared with that in untrained rats (SCI control).

following SCI, which may not be sensitive to the BBB scale. While walking on a horizontal ladder, the alternating activity pattern of antagonistic leg muscles and the coordination between legs is orchestrated by the CPG; the CPG networks are activated by tonic input from the reticular formation in the brainstem. ${ }^{19}$ However, this input was almost cutoff or seriously reduced after SCI in our model; therefore, the activation of CPG should be elicited by a different input derived from hind limb stepping or walking induced by TANES. We suggest that the restoration of hind limb sensorimotor function is, in all likelihood, attributable to the activity-dependent plasticity that results in the acquisition and maintenance of motor skills.
Results from this study showed that H-latency, M-latency, H-amplitude, $\mathrm{M}$-amplitude and $\mathrm{H} / \mathrm{M}$ amplitude ratio were seriously affected by SCI, but later showed limited and slow recovery. Importantly, the $\mathrm{H} / \mathrm{M}$ amplitude ratio increased because of the increase in $\mathrm{H}$-amplitude and the decrease in $\mathrm{M}$-amplitude. The increase in the $\mathrm{H}$-wave amplitude after SCI reinforces the presynaptic noninhibition theory. ${ }^{20}$ We found that TANES-induced walking training significantly improved the restoration of $\mathrm{H} / \mathrm{M}$ amplitude ratio, revealing that TANES or TANES-induced walking training may benefit the recovery of supraspinal control or H-reflex. ${ }^{21}$ These results agreed with other studies showing that passive exercise helps recover the 
frequency-dependent depression of the H-reflex in animals and humans after SCI. ${ }^{22}$ The mechanisms remain unclear.

\section{CONCLUSION}

Early application of TANES-induced walking training significantly promotes locomotor recovery in rats with spinal cord contusion injury without other treatments such as cell/tissue transplantation. The restoration of locomotion may be related to the activity-dependent plasticity below the injury level through the activation of $\mathrm{CPG}$ triggered by TANES. TANES-induced walking training may also cause improvement in electrophysiological restoration such as the H-reflex. However, the mechanisms that are associated with activity-dependent plasticity require more understanding.

\section{DATA ARCHIVING}

There were no data to deposit.

\section{CONFLICT OF INTEREST}

The authors declare no conflict of interest.

\section{ACKNOWLEDGEMENTS}

This study was supported by the Spinal Cord Society and partially by Natural Science Foundation of China (NSFC).

1 Molinari M. Plasticity properties of CPG circuits in humans: impact on gait recovery. Brain Res Bull 2009; 78: 22-25.

2 Van de Crommert HW, Mulder T, Duysens J. Neural control of locomotion: sensory control of the central pattern generator and its relation to treadmill training. Gait Posture 1998; 7: 251-263.

3 Dunlop SA. Activity-dependent plasticity: implications for recovery after spinal cord injury. Trends Neurosci 2008; 31: 410-418.

4 Ichiyama R, Potuzak M, Balak M, Kalderon N, Edgerton VR. Enhanced motor function by training in spinal cord contused rats following radiation therapy. PLOS ONE 2009; 4: e6862.

5 Anwer S, Equebal A, Palekar TJ, Nezamuddin M, Neyaz O, Alghadir A. Effect of locomotor training on motor recovery and walking ability in patients with incomplete spinal cord injury: a case series. J Phys Ther Sci 2014; 26: 951-953.

6 Fakhoury M. Spinal cord injury: overview of experimental approaches used to restore locomotor activity. Rev Neurosci 2015; 26: 397-405.

7 Field-Fote EC. Spinal cord control of movement: implications for locomotor rehabilitation following spinal cord injury. Phys Ther 2000; 80: 477-484.

8 Zhang SX, Huang F, Gates M, Holmberg EG. Tail nerve electrical stimulation combined with scar ablation and neural transplantation promotes locomotor recovery in rats with chronically contused spinal cord. Brain Res 2012; 1456: 22-35.
9 Magnuson DS, Lovett R, Coffee C, Gray R, Han Y, Zhang YP et al. Functional consequences of lumbar spinal cord contusion injuries in the adult rat. J Neurotrauma 2005; 22: 529-543.

10 Zhang SX, Huang F, Gates M, Holmberg EG. Somatosensory evoked potentials can be recorded on the midline of the skull with subdermal electrodes in non-sedated rats elicited by magnetic stimulation of the tibial nerve. J Neurosci Methods 2012; 208: 114-118.

11 Basso DM, Beattie MS, Bresnahan JC. Graded histological and locomotor outcomes after spinal cord contusion using the NYU weight-drop device versus transection. Exp Neurol 1996; 139: 244-256.

12 Metz GA, Whishaw IQ. The ladder rung walking task: a scoring system and its practical application. J Vis Exp 2009; 28: 1-4.

13 Loy DN, Magnuson DS, Zhang YP, Onifer SM, Mills MD, Cao QL et al. Functional redundancy of ventral spinal locomotor pathways. J Neurosci 2002; 22: 315-323.

14 Zhang YP, Shields LB, Zhang Y, Pei J, Xu XM, Hoskins $R$ et al. Use of magnetic stimulation to elicit motor evoked potentials, somatosensory evoked potentials, and H-reflexes in non-sedated rodents. J Neurosci Methods 2007; 165: 9-17.

15 Grillner S. The motor infrastructure: from ion channels to neuronal networks. Nat Rev Neurosci 2003; 4: 573-586.

16 Harkema S, Gerasimenko Y, Hodes J, Burdick J, Angeli C, Chen Y et al. Effect of epidural stimulation of the lumbosacral spinal cord on voluntary movement, standing, and assisted stepping after motor complete paraplegia: a case study. Lancet 2011; 377: 1938-1947.

17 Wolpaw JR, Tennissen AM. Activity-dependent spinal cord plasticity in health and disease. Annu Rev Neurosci 2001; 24: 807-843.

18 McEwen ML, Springer JE. Quantification of locomotor recovery following spinal cord contusion in adult rats. J Neurotrauma 2006; 23: 1632-1653.

19 Bolton DA, Tse AD, Ballermann M, Misiaszek JE, Fouad K. Task specific adaptations in rat locomotion: runway versus horizontal ladder. Behav Brain Res 2006; 168: 272-279.

20 Ollivier-Lanvin K, Keeler BE, Siegfried R, Houle JD, Lemay MA. Proprioceptive neuropathy affects normalization of the $\mathrm{H}$-reflex by exercise after spinal cord injury. Exp Neurol 2010; 221: 198-205.

21 Knikou M, Mummidisetty CK. Locomotor training improves premotoneuronal control after chronic spinal cord injury. J Neurophysiol 2014; 111: 2264-2275.

22 Reese NB, Skinner RD, Mitchell D, Yates C, Barnes CN, Kiser TS et al. Restoration of frequency-dependent depression of the $\mathrm{H}$-reflex by passive exercise in spinal rats. Spinal Cord 2006; 44: 28-34.

(c) (i) (2) This work is licensed under a Creative Commons Ay NC SA Attribution-NonCommercial-ShareAlike 4.0

International License. The images or other third party material in this article are included in the article's Creative Commons license, unless indicated otherwise in the credit line; if the material is not included under the Creative Commons license, users will need to obtain permission from the license holder to reproduce the material. To view a copy of this license, visit http://creativecommons.org/ licenses/by-nc-sa/4.0/

Supplementary Information accompanies this paper on the Spinal Cord website (http://www.nature.com/sc) 